\title{
AUTHOR INDEX \\ Volume 5
}

Ahmadian, M. T., see Mojahedi, M.

3 (2013) 1350032

Asadi, H., Javaherdeh, K. \& Ramezani, S., Finite Element

Simulation of Micropolar Fluid Flow in the Lid-Driven

Square Cavity

4 (2013) 1350045

Asadi, H., Javaherdeh, K. \& Ramezani, S., Micropolar Fluid

Model for Blood Flow Through a Stenosed Artery

Bai, S.-L., see Huang, Z.

Bai, Z., see Meng, X.

Bouchaala, N., Dion, J. L., Peyret, N. \& Haddar, M.,

Micro-Slip Induced Damping in the Contact of Nominally

Flat Surfaces

1 (2013) 1350005

Bui, T. Q., see Valizadeh, N.

Chakrabarti, A., see Taj, M. N. A. G.

2 (2013) 1350017

4 (2013) 1350041

Chao, Y. J., see Wang, Z.-X.

3 (2013) 1350029

Chen, L. \& Wei, Y., Characterizations of Size Effect and

Overall Mechanical Behavior of Nanocrystalline Metals

Chen, Y., see Huang, Z.

Cheng, J., see Dai, B.

4 (2013) 1350043

3 (2013) 1350035

4 (2013) 1350044

Cheng, Y., Zhang, Z. \& Teo, Z., Deformation of Graphene

Induced by Adsorption of Peptides: A Molecular Dynamics Study

Dai, B., Cheng, J. \& Zheng, B., A Moving Kriging

Interpolation-Based Meshless Local Petrov-Galerkin

Method for Elastodynamic Analysis

1 (2013) 1350004

3 (2013) 1350035

1 (2013) 1350011

Daining, F., see Yiping, L.

Davis, J. \& Ramulu, M., A Semi-Analytical Model of Time

Dependent Plastic Strains Induced During Shot Peening

Deng, Z., see $\mathrm{Hu}, \mathrm{W}$.

1 (2013) 1350007

Ding, H. \& Zu, J. W., Periodic and Chaotic Responses of an Axially Accelerating Viscoelastic Beam Under

Two-Frequency Excitations

2 (2013) 1350019

Dion, J. L., see Bouchaala, N.

1 (2013) 1350005

Dion, J. L., see Jrad, H.

2 (2013) 1350018 
Fan, L. F., Yi, X. W. \& Ma, G. W., Numerical Manifold Method (NMM) Simulation of Stress Wave Propagation Through Fractured Rock Mass

Fan, X., see Suo, T.

Fan, Z., Lu, G., Yu, T. X. \& Liu, K., Axial Crushing of

Triangular Tubes

2 (2013) 1350022

2 (2013) 1350016

Fang, D., see Wang, R.

Fang, D., see Zhou, W.

Feng, X.-Q., see Zhao, H.-P.

Firoozbakhsh, K., see Mojahedi, M.

Freund, J., see Karakoç, A.

Freund, J., see Karakoç, A.

1 (2013) 1350008

3 (2013) 1350026

4 (2013) 1350040

2 (2013) 1350012

3 (2013) 1350032

2 (2013) 1350021

3 (2013) 1350034

Gad, E., see Yang, Y.

Ghugal, Y. M. \& Sayyad, A. S., Stress Analysis of Thick

2 (2013) 1350013

Laminated Plates Using Trigonometric Shear Deformation

Theory

Going, J., see Li, W. G.

1 (2013) 1350003

3 (2013) 1350024

Haddar, M., see Bouchaala, N.

1 (2013) 1350005

Haddar, M., see Jrad, H.

2 (2013) 1350018

Hao, F., see Zhou, W.

4 (2013) 1350040

Hill, N. A., see Li, W. G.

3 (2013) 1350024

Hong, W., see Toh, W.

1 (2013) 1350001

$\mathrm{Hu}, \mathrm{G}$., see Shen, Z.

3 (2013) 1350031

Hu, W., Deng, Z. \& Ouyang, H., Generalized

Multi-Symplectic Method for Dynamic Responses of

Continuous Beam Under Moving Load

3 (2013) 1350033

3 (2013) 1350030

$\mathrm{Hu}$, Z., see Tang, H.

Huang, L. \& Liao, H., Nonlinear Aerodynamic Forces on the

Flat Plate in Large Amplitude Oscillation

4 (2013) 1350039

Huang, Z., Chen, Y. \& Bai, S.-L., An Elastoplastic

Constitutive Model for Porous Materials

3 (2013) 1350035

Jacob, C., Sepahvand, K., Matsagar, V. A. \& Marburg, S.,

Stochastic Seismic Response of Base-Isolated Buildings

1 (2013) 1350006

Javaherdeh, K., see Asadi, H.

4 (2013) 1350043

Javaherdeh, K., see Asadi, H.

4 (2013) 1350045

Jrad, H., Renaud, F., Dion, J. L., Tawfiq, I. \& Haddar, M.,

Experimental Characterization, Modeling and Parametric

Identification of the Hysteretic Friction Behavior of

Viscoelastic Joints

Kalantari, M., see Shen, J. J.

2 (2013) 1350018

3 (2013) 1350028

Karakoç, A. \& Freund, J., A Direct Simulation Method for the Effective In-Plane Stiffness of Cellular Materials

3 (2013) 1350034 
Karakoç, A. \& Freund, J., Statistical Strength Analysis for Honeycomb Materials

2 (2013) 1350021

Lam, N., see Yang, Y.

Lam, P.-S., see Wang, Z.-X.

2 (2013) 1350013

Li, B.-W., see Zhao, H.-P.

Li, C. G., see Shen, J. J.

3 (2013) 1350029

2 (2013) 1350012

Li, M., see Meng, X.

Li, W. G., Going, J., Hill, N. A. \& Luo, X. Y., Breaking

3 (2013) 1350028

4 (2013) 1350044

Analysis of Artificial Elastic Tubes and Human Artery

Li, W., see Wang, R.

3 (2013) 1350024

Li, X., see Tang, H.

3 (2013) 1350026

3 (2013) 1350030

Li, Y., see Suo, T.

2 (2013) 1350016

Liao, H., see Huang, L.

4 (2013) 1350039

Lim, C. W., see Sun, J.

4 (2013) 1350042

Liqun, T., see Yiping, L.

1 (2013) 1350009

Liu, D., see Wang, Y.

2 (2013) 1350023

Liu, K., see Fan, Z.

1 (2013) 1350008

Liu, Z., see Toh, W.

1 (2013) 1350001

Lu, G., see Fan, Z.

1 (2013) 1350008

Luo, X. Y., see Li, W. G.

3 (2013) 1350024

Luo, X. Y., see Wu, B.

1 (2013) 1350002

Ma, G. W., see Fan, L. F.

2 (2013) 1350022

1 (2013) 1350006

Marburg, S., see Jacob, C.

1 (2013) 1350006

Meng, X., Bai, Z. \& Li, M., Elastodynamic Stress Intensity

Factor for a Finite Crack in Elastic Solid Under 3D

Transient Loading of Mode I

Ming, L., see Suo, T.

Mojahedi, M., Ahmadian, M. T. \& Firoozbakhsh, K.,

Dynamic Pull-In Instability and Vibration Analysis of a

Nonlinear Microcantilever Gyroscope Under Step Voltage

Considering Squeeze Film Damping

3 (2013) 1350032

Nayebi, A., see Yazdani, H.

4 (2013) 1350038

Ng, T. Y., see Toh, W.

1 (2013) 1350001

Nguyen, M. N., see Valizadeh, N.

2 (2013) 1350017

Ouyang, H., see $\mathrm{Hu}$, W.

3 (2013) 1350033

Panoskaltsis, V. P. \& Soldatos, D., A Phenomenological

Constitutive Model of Non-Conventional Elastic Response

4 (2013) 1350036

Peyret, N., see Bouchaala, N.

1 (2013) 1350005

Qin, Q., Wang, M., Wang, Z., Zhang, J. \& Wang, T. J., A

Yield Criterion and Plastic Analysis for Physically

Asymmetric Sandwich Beam with Metal Foam Core

4 (2013) 1350037 
Ramezani, S., see Asadi, H.

4 (2013) 1350043

Ramezani, S., see Asadi, H.

4 (2013) 1350045

Ramulu, M., see Davis, J.

3 (2013) 1350027

Rana, M., see Shahid, N.

1 (2013) 1350010

Renaud, F., see Jrad, H.

2 (2013) 1350018

Saif, T. A., see Tang, X.

3 (2013) 1350025

Sayyad, A. S., see Ghugal, Y. M.

1 (2013) 1350003

Sepahvand, K., see Jacob, C.

1 (2013) 1350006

Shahid, N. \& Rana, M., The Influence of Deborah Number on Some Couette Flows of a Maxwell Fluid

1 (2013) 1350010

Shen, J. J., Li, C. G., Wu, H. T. \& Kalantari, M., The

Frictional Contact Analysis Between a Tactile Sensor and Atrial Tissue in Viscoelasticity

Shen, Z. \& Hu, G., Thermally Induced Vibrations of Solar

Panel and Their Coupling with Satellite

Shengping, S., see $\mathrm{Xu}$, L.

Shigang, A., see Yiping, L.

Soldatos, D., see Panoskaltsis, V. P.

Sun, J., Xu, X. \& Lim, C. W., Symplectic Method for

Dynamic Buckling of Cylindrical Shells Under Combined

Loadings

4 (2013) 1350042

Suo, T., Ming, L., Zhao, F., Li, Y. \& Fan, X., Temperature and Strain Rate Sensitivity of Ultrafine-Grained Copper Under Uniaxial Compression

Taj, M. N. A. G. \& Chakrabarti, A., Buckling Analysis of

Functionally Graded Skew Plates: An Efficient Finite

Element Approach

3 (2013) 1350028

3 (2013) 1350031

2 (2013) 1350015

1 (2013) 1350009

4 (2013) 1350036

Tang, H., Hu, Z. \& Li, X., Three-Dimensional

Pressure-Dependent Elastoplastic Cosserat Continuum

Model and Finite Element Simulation of Strain

Localization

3 (2013) 1350030

Tang, X. \& Saif, T. A., Adhesivity of Colon Cancer Cells

During In Vitro Metastasis

3 (2013) 1350025

2 (2013) 1350018

Tawfiq, I., see Jrad, H.

Teo, Z., see Cheng, Y.

1 (2013) 1350007

Thai, H. T., see Valizadeh, N.

Tinghui, H., see Yiping, L.

2 (2013) 1350017

1 (2013) 1350009

Toh, W., Liu, Z., Ng, T. Y. \& Hong, W., Inhomogeneous

Large Deformation Kinetics of Polymeric Gels

1 (2013) 1350001

Valizadeh, N., Bui, T. Q., Vu, V. T., Thai, H. T. \& Nguyen,

M. N., Isogeometric Simulation for Buckling, Free and

Forced Vibration of Orthotropic Plates

2 (2013) 1350017 
Van Hirtum, A., see $\mathrm{Wu}, \mathrm{B}$.

1 (2013) 1350002

$\mathrm{Vu}, \mathrm{V}$. T., see Valizadeh, N.

2 (2013) 1350017

Wang, M., see Qin, Q.

4 (2013) 1350037

Wang, R., Li, W. \& Fang, D., A Thermo-Damage Strength

Model for the SiC-Depleted Layer of

Ultra-High-Temperature Ceramics on High Temperature

Oxidation

3 (2013) 1350026

Wang, T. J., see Qin, Q.

Wang, Y., Zhang, X. \& Liu, D., Asymptotic Analysis of

4 (2013) 1350037

Generalized Thermoelasticity for Axisymmetric Plane

Strain Problem with Temperature-Dependent Material

Properties

Wang, Y., see Zhao, H.-P.

Wang, Z., see Qin, Q.

Wang, Z.-X., Zhang, R.-F., Chao, Y. J. \& Lam, P.-S., Effect

of Mechanical Property Mismatch on Failure Assessment

Curve for Welded Joint with a Semi-Elliptical Crack

3 (2013) 1350029

1 (2013) 1350004

4 (2013) 1350046

Wei, Z., see Zheng, H.

Wu, B., Van Hirtum, A. \& Luo, X. Y., Pressure Driven

Steady Flow in Constricted Channels of Different

Cross-Section Shapes

2 (2013) 1350023

2 (2013) 1350012

4 (2013) 1350037

Wu, H. T., see Shen, J. J.

Xu, L. \& Shengping, S., Size-Dependent Piezoelectricity and

Elasticity Due to the Electric Field-Strain Gradient

Coupling and Strain Gradient Elasticity

$\mathrm{Xu}, \mathrm{X}$., see Sun, J.

Yang, Y., Lam, N., Zhang, L. \& Gad, E., Simplified Analysis of Low Velocity Impact Actions on Shallow Domes

1 (2013) 1350002

3 (2013) 1350028

Yazdani, H. \& Nayebi, A., Continuum Damage Mechanics Analysis of Thin-Walled Tube Under Cyclic Bending and Internal Constant Pressure

Yi, X. W., see Fan, L. F.

Yiping, L., Liqun, T., Shigang, A., Zejia, L., Tinghui, H., Zhenyu, J. \& Daining, F., Design and Mechanical

Properties of Liquid Rubber-Based Concrete

1 (2013) 1350009

Yu, T. X., see Fan, Z.

1 (2013) 1350008

Yuan, X. \& Zhu, Z. H., Wave Reflection in Piezoelectric Half-Plane

2 (2013) 1350014

1 (2013) 1350009

Zenkour, A. M., Bending of FGM Plates by a Simplified Four-Unknown Shear and Normal Deformations Theory

2 (2013) 1350020 
Zhang, J., see Qin, Q.

Zhang, L., see Yang, Y.

Zhang, R.-F., see Wang, Z.-X.

Zhang, X., see Wang, Y.

Zhang, Z., see Cheng, Y.

Zhao, F., see Suo, T.

Zhao, H.-P., Wang, Y., Li, B.-W. \& Feng, X.-Q.,

Improvement of the Peeling Strength of Thin Films by a

Bioinspired Hierarchical Interface

Zheng, B., see Dai, B.

Zheng, H. \& Wei, Z., Vibroacoustic Analysis of Stiffened

Plates with Nonuniform Boundary Conditions

Zhenyu, J., see Yiping, L.

Zhou, W., Hao, F. \& Fang, D., The Effects of Elastic

Stiffening on the Evolution of the Stress Field within a

Spherical Electrode Particle of Lithium-Ion Batteries

Zhu, Z. H., see Yuan, X.

$\mathrm{Zu}$, J. W., see Ding, H.
4 (2013) 1350037

2 (2013) 1350013

3 (2013) 1350029

2 (2013) 1350023

1 (2013) 1350007

2 (2013) 1350016

2 (2013) 1350012

1 (2013) 1350011

4 (2013) 1350046

1 (2013) 1350009

4 (2013) 1350040

2 (2013) 1350014

2 (2013) 1350019 